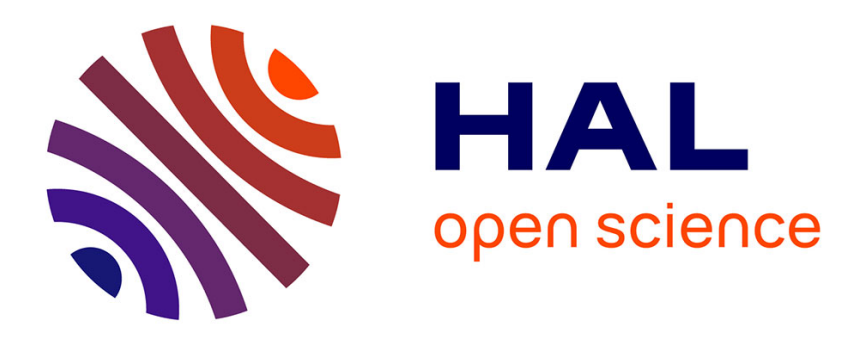

\title{
La dragonnade du Poitou et l'exil des huguenots dans la littérature de controverse anglaise
}

Anne Dunan-Page

\section{To cite this version:}

Anne Dunan-Page. La dragonnade du Poitou et l'exil des huguenots dans la littérature de controverse anglaise. Moreana, 2007, 171-172, pp.86-121. halshs-00839776

\section{HAL Id: halshs-00839776 \\ https://shs.hal.science/halshs-00839776}

Submitted on 30 Jun 2013

HAL is a multi-disciplinary open access archive for the deposit and dissemination of scientific research documents, whether they are published or not. The documents may come from teaching and research institutions in France or abroad, or from public or private research centers.
L'archive ouverte pluridisciplinaire HAL, est destinée au dépôt et à la diffusion de documents scientifiques de niveau recherche, publiés ou non, émanant des établissements d'enseignement et de recherche français ou étrangers, des laboratoires publics ou privés. 


\title{
La dragonnade du Poitou et l'exil des huguenots dans la littérature de controverse anglaise ${ }^{1}$
}

\author{
Anne Dunan-Page
}

'Nous sommes devenus le spectacle de la terre par nos calamitez'

Paul Fétizon, Apologie des Reformez (1683)

Le 21 juillet 1681, après avoir écouté en son conseil le récit d'une députation des deux églises françaises de Londres (Threadneedle et La Savoie) le roi Charles II décida de rédiger puis de faire imprimer deux lettres qui, accompagnées par l'Ordre d'Hampton Court du 28 juillet et le Bref du 10 septembre, allaient décider du sort des huguenots exilés en Angleterre. La première lettre était destinée à l'évêque de Londres, Henry Compton, la seconde au maire, le Whig anticatholique Patience Ward ${ }^{2}$. Le roi y rappelle tout d'abord sa compassion pour les Français, non pas en tant qu'étrangers dans le besoin mais en tant que protestants persécutés: 'We being touched with a true sense and compassion of their deplorable condition, and looking upon them not only as distressed Strangers, but chiefly as persecuted Protestants'. Ces missives avaient ensuite pour but d'encourager officiellement les autorités ecclésiastiques et municipales à accueillir et à soutenir financièrement la 'Sainte Colonie de protestants de France' qui, selon la rumeur, arrivaient chaque semaine en haillons, affamés, terrorisés, ayant quitté famille, logis et patrie sous la pression des dragons ${ }^{3}$. Selon un récit contemporain, ils auraient été ainsi quelque 60000 à 80000 à quitter la France depuis les années $1660^{4}$. Il y a déjà près de trente ans, Robin

\footnotetext{
${ }^{1}$ L'auteur tient à exprimer sa plus vive reconnaissance à Luc Borot et à Yves Krumenacker, ainsi qu'aux personnels de la Bibliothèque de la Société de l'Histoire du Protestantisme Français, la Bibliothèque Nationale de France, The British Library, The Mumby Rare Books Room (Cambridge University Library), Duke Humphrey's Library (Bodleian Library), St John's College Library (Cambridge) et The Wren Library, Trinity College (Cambridge). Les recherches ont été effectuées pendant un congé attribué par la $11^{\mathrm{e}}$ section du Conseil National des Universités (C.N.U).

${ }^{2}$ Voir His Majesties Letters to the Bishop of London and the Lord Mayor datées du 22 juillet 1681 et Calendar of State Papers, Domestic, 22 juillet 1681 (ci-après cités CSPD). Un autre document des CSPD semble indiquer que les lettres ne furent en fait imprimées que le 28 juillet. Pour l'immigration huguenote après 1680, voir Bernard Cottret, The Huguenots in England: Immigration and Settlement c.1550-1700, trad. Peregrine et Adriana Stevenson (1985; Cambridge, 1991), pp. 185-204. Je cite la traduction anglaise, révisée par l'auteur; Robin Gwynn, 'Arrival of Huguenot Refugees in England, 1680-1705', Proceedings of the Huguenot Society of London, 21/4 (1969), pp. 366373 (ci-après notés PHS); Robin Gwynn, Huguenot Heritage: The History and Contribution of the Huguenots in

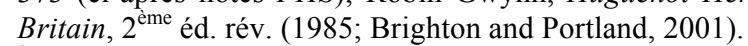

${ }^{3}$ [André Lombard], Harangue au Roy : Faite par un Ministre de l'Eglise Francoise de la Savoye (Londres, 1681), p. 3. Voir également The True Impartiall Mercury, $\mathrm{n}^{\circ} 48$ (du 4 au 7 octobre 1681) et pour des références à l'arrivée des huguenots, CSPD (11 août 1681, 3 septembre 1681, 13 septembre 1681, 19 septembre 1681).

${ }^{4}$ Pierre Jurieu, La Politique du Clergé de France ou Entretiens Curieux de deux Catholiques Romains, $2^{\text {ème }}$ éd. (La Haye, 1681), p. 199. Voir R.J. Howells, Pierre Jurieu: Antinominan Radical (Durham, 1983), pp. 29-43 et Jacques
} 
Gwynn, dans une étude croisée des livres de témoignages et de reconnaissances des églises françaises de Londres, a pu établir que pour la seule année 1681, ce sont 1182 protestants exilés qui ont été recensés 5 .

Si l'impact démographique, religieux, politique et culturel de la Révocation de l'Édit de Nantes en Angleterre est aujourd'hui bien connu des chercheurs, celui de la dragonnade du Poitou de 1681 l'est bien moins ${ }^{6}$. Le but de cet article est d'abord de révéler la multiplicité des points de vue sur l'exil huguenot, en particulier dans la presse d'information et dans la littérature de controverse. Nous montrerons ensuite comment, après la dissolution du dernier parlement du règne de Charles II en mars 1681, le parti Whig saisit l'occasion des violences poitevines pour raviver la querelle sur les possibles effets de la succession d'un roi catholique sur le trône d'Angleterre. Nous envisagerons les événements français de l'été et de l'automne 1681 comme un moment décisif dans la lutte pour le contrôle de la presse entre les partis Whig et Tory, lutte dans laquelle les huguenots furent malgré eux impliqués. Il nous faudra d'autre part garder à l'esprit que les Anglais connaissaient mal ces exilés. Les informations relayées par les imprimés de grande diffusion, par les journaux en particulier, étaient donc essentielles pour représenter ceux qu'on appelait hugonots, hugonotes, hugonets, hugenots, hugenotes, la variété orthographique trahissant certes l'absence de stabilité de la langue écrite du XVIIe siècle, mais aussi les hésitations (qui perdurent dans les pays anglo-saxons) sur la prononciation même du mot.

\section{Juillet 1681 : l'exil dans l'urgence}

Point n'est ici besoin de rappeler les détails de la dragonnade de 1681 aujourd'hui bien documentée par les travaux essentiels d'Élisabeth Labrousse, Janine Garrisson et Yves Krumenacker $^{7}$. La dragonnade, limitée au seul Poitou, n'est guère comparable à celle de 1685

Solé, 'La diplomatie de Louis XIV et les protestants français réfugiés aux Provinces-Unies (1678-1688)', Bulletin de la Société de l'Histoire du Protestantisme Français, 115 (octobre-novembre1969), pp. 627-60. Ci-après noté BSHPF. ${ }^{5}$ Gwynn, 'Arrival of Huguenot Refugees', p. 373.

${ }^{6}$ Pour la révocation, voir John Miller, 'The Immediate Impact of the Revocation in England', dans C.E.J. Caldicott, H. Gough et Jean-Paul Pittion (eds), The Huguenots in Ireland: Anatomy of an Emigration (Dun Laoghaire, 1987), pp. 161-203; John Marshall, 'Huguenot Thought after the Revocation of the Edict of Nantes: Toleration, "Socinianism", Integration and Locke', dans Randolph Vigne and Charles Littleton (eds), From Strangers to Citizens: The Integration of Immigrant Communities in Britain, Ireland and Colonial America, 1550-1750 (Brighton and Portland, 2001), pp. 383-396; Gwynn, Huguenot Heritage, pp. 166-182.

${ }^{7}$ Élisabeth Labrousse, 'Une foi, une loi, un roi?' La Révocation de L'Édit de Nantes (Genève et Paris, 1985), pp. 167-177 ; Janine Garrisson, L'Édit de Nantes et sa Révocation. Histoire d'une intolérance (Paris, 1985), pp. 127183 ; Yves Krumenacker, 'Les dragonnades du Poitou: leur écho dans les mémoires', BSHPF, 131 (juillet-août 1985), pp. 405-422. Voir également Jean Orcibal, Louis XIV et les Protestants (Paris, 1951), pp. 69-77 ; Samuel Mours, Le Protestantisme en France au XVIIe siècle (1598-1685) (Paris, 1967), pp. 159-163 ; Robert Poujol, 'Le rôle des intendants dans les préliminaires de la Révocation', in Roger Zuber et Laurent Theis (eds), La Révocation de l'Édit de Nantes et le protestantisme français en 1685 (Paris, 1986), pp. 87-112. Krumenacker reprend et développe 
(qui par son étendue et sa violence fut un rude prélude à la Révocation de l'Edit de Nantes) mais elle choqua les esprits par son intensité et par les parallèles qu'on pouvait dès lors établir entre les situations anglaise et française. Par lettre du 18 mars 1681, l'intendant du Poitou, René de Marillac obtint du Ministre de la Guerre Louvois l'envoi des dragons ${ }^{8}$. Marillac semble même avoir anticipé les directives du ministre, l'arrivée des troupes n'ayant été à l'origine prévue que pour novembre. Une fois logés chez les protestants (les 'nouveaux convertis' ayant déjà été exemptés du logement des troupes par l'ordonnance du 11 avril 1681), ${ }^{9}$ les dragons avaient loisir de se faire payer, nourrir et servir, puis de commettre toutes sortes d'exactions pour obtenir l'abjuration de leurs hôtes. Les récits parlent de personnes maltraitées, battues, 'chauffées' (c'est-à-dire maintenues à proximité immédiate d'un feu), comme la femme de Jean Migault ou 'Pierre Bonneau' mentionné par Élie Benoist, de vieillards attachés à des bancs, d'enfants terrorisés et violentés ${ }^{10}$. Jurieu ajoute que les soldats n'hésitaient pas à brûler ou à frapper la plante des pieds, à 'serrer les pouces', à pendre les femmes au plafond, à les forcer à ingurgiter de l'eau par un entonnoir, à placer des pierres dans la bouche pour 'aiguiser les dents ${ }^{11}$. Benoist, quant à lui, insiste sur la privation de sommeil, une technique particulièrement efficace, souvent utilisée pour soutirer aux sorcières la confession de leurs méfaits ${ }^{12}$. L'horreur s'arrêtait théoriquement au viol et au meurtre. Si des pertes humaines furent à déplorer, les auteurs en firent généralement les conséquences 'involontaires' des tortures qui devaient faire souffrir pour amener à l'abjuration mais sans entraîner la mort. Une fois les habitants battus et ruinés, leur demeure pouvait être démantelée, les meubles et autres effets personnels vendus pour une ultime chope de bière, les huguenots réfractaires devant subir l'ultime humiliation de voir tel meuble de famille trôner dans la salle d'un voisin ou une 'nouvelle convertie' se pavaner avec les robes d'une parente plus difficile à convaincre ${ }^{13}$.

Les violences physiques, internes et externes, les purifications par l'eau et par le feu, étaient ainsi accompagnées de violences sur les biens, tout aussi craintes des habitants pour qui

ses thèses dans Les Protestants du Poitou au XVIIIe siècle (1681-1789) (Paris, 1998), pp. 53-132 (pour la dragonnade, voir pp. 62-83).

${ }^{8}$ Voir par exemple, Poujol, 'Le rôle des intendants', p. 100.

${ }^{9}$ Pour l'ordonnance, voir Edits, Déclarations et Arrests concernans la Réligion P. Réformée, 1662-1751, ed. Léon Pilatte (Paris, 1885), pp. 79-80.

${ }^{10}$ Voir Jean Migault, Journal de Jean Migault ou Malheurs d'une famille protestante du Poitou victime de la Révocation de l'Édit de Nantes, 1682-1689, ed. Yves Krumenacker (Paris, 1995), pp. 27-45; Élie Benoist, Histoire de l'Édit de Nantes, t. IV (Delft, 1695), pp. 478-483 (p. 482 pour Bonneau). Voir aussi 'une dragonnade en Poitou en 1681', BSHPF 52 (1903), pp. 256-268 pour le récit d'Abraham Papot et Jurieu, Les Derniers Efforts de L'Innocence Affligée (La Haye, 1682), I, pp. 110-182 (ci-après noté I pour le premier entretien et II pour le second, qui sont paginés séparément dans l'édition consultée). Pour Jurieu et la dragonnade, voir Guy Howard Dodge, The Political Theory of the Huguenots of the Dispersion. With Special Reference to the Thought and Influence of Pierre Jurieu (New York, 1947), pp. 12-33.

${ }^{11}$ Jurieu, Derniers Efforts, I, pp. 120, 163.

${ }^{12}$ Benoist, t. IV, p. 482.

${ }^{13}$ Jurieu, Derniers Efforts, I, pp. 36, 40, 44. 
s'ensuivait une ruine certaine. Chaque cas de maltraitance était en outre assorti du spectacle des compensations financières et matérielles qu'engendrait l'abjuration. Au total, on estime que ce sont entre 30000 et 40000 abjurations que les autorités purent se flatter d'avoir enregistrées dans la région entre mai et novembre. ${ }^{14}$ Comme le fait remarquer Jurieu avec une ironie mordante, c'était bien la constitution d'une Église de 'scélérats' qui se préparait si la France entière comptait suivre l'exemple du Poitou en faisant 'des Saints en un quart d'heure', 'la bourse dans une main, et les armes dans l'autre' ${ }^{15}$.

La dragonnade du Poitou, contrepoint d'une répression plus 'en douceur' perpétrée à coups d'édits, de déclarations, et de démolition des lieux de culte, ne pouvait que susciter l'émoi des protestants étrangers ${ }^{16}$. Quelques jours après ses lettres destinées à Compton et à Ward, Charles II prononça en conseil l'Ordre d'Hampton Court dans lequel il promit notamment la naturalisation des protestants français par le prochain parlement, une denization gratuite et immédiate pour tous les réfugiés qui en feraient la demande, et les mêmes droits à l'éducation pour les enfants français que pour les enfants anglais. La déclaration de juillet qui abaissait à sept ans l'âge des conversions en France avait eu un impact dramatique. On possède également la preuve, grâce à une lettre du Secrétaire d'État Leoline Jenkins, qu'à la fin juillet, Jenkins cherchait des précédents dans les archives de son ministère qui permettraient à Charles II d'intervenir directement auprès de Louis XIV ${ }^{17}$.

Le 10 septembre, la collecte londonienne prévue dans les lettres de juillet fut élargie par Bref à l'ensemble du pays, avec le succès que l'on sait ${ }^{18}$. En dernier lieu, un comité se réunissant chaque semaine, voire plus si nécessaire, fut mis en place pour superviser la distribution des aides. Il était composé de William Sancroft (archevêque de Cantorbéry), Henry Compton

\footnotetext{
${ }^{14}$ Krumenacker, Protestants du Poitou, p. 79.

${ }^{15}$ Jurieu, Derniers Efforts, I, pp. 110, 112, 115.

${ }^{16}$ Pour une analyse méthodique des moyens de répression voir Jurieu, Politique du Clergé, pp. 21-105. Jurieu rapporte les édits qui restreignent l'exercice du culte, ceux qui sont destinés à faire abandonner la religion, et finalement les différentes techniques d' 'adoucissement' (pp. 21-105). L'historiographie contemporaine tente aussi souvent d'ordonner les nombreux arrêts, déclarations et édits de la période. Voir par exemple Mours (qui distingue quatre tendances : propagande, séduction, réunion et contrainte) ; Garrisson, et Krumenacker, Protestants du Poitou, pp. 53-59 (qui en distinguent trois : empêcher le groupe protestant de se désigner comme tel, empêcher que ne se perpétue la religion protestante, contribuer à son élimination). Krumenacker démontre les erreurs de jugement des autorités qui pensaient que l'anéantissement du protestantisme passait par la conversion des élites et la suppression du culte.

${ }_{17}^{17}$ Savile Correspondence, ed. William Durrant Cooper (Londres, 1858), p. 214.

18 'A Brief for the Persecuted Protestants of France' est reproduit par George B. Beeman, 'Notes on the City of London Records Dealing with the French Protestant Refugees, Especially with Reference to the Collections Made under Various Briefs', PHS, 7 (1901-1904), pp. 108-92. Voir aussi, Irene Scouloudi, 'L'aide apportée aux réfugiés protestants français par l'Église de Threadneedle Street, l'Église de Londres, 1681-1687', BSHPF 115 (1969), pp. 429-444; A.P. Hands et Irene Scouloudi, French Protestant Refugees Relieved through the Threadneedle Street Church, London, 1681-1687, Huguenot Society of London, Quarto Series XLIX (Londres, 1971), pp. 1-3 ; Sugiko Nishikawa, 'English Attitudes towards Continental Protestants with Particular Reference to Church Briefs, c. 16801740', thèse de doctorat non publiée (Londres, 1998). Pour un point de vue contemporain, voir Narcissus Luttrell, $A$ Brief Historical Relation of State Affairs from September 1678 to April 1714, vol. 1 (Oxford, 1857), pp. 113, 125$126,133$.
} 
(évêque de Londres), Edward Stillingfleet (doyen de la Cathédrale St Paul), Sir Patience Ward (maire de la capitale), Thomas Papillon et Jean Dubois (marchand et tisserand de leur état et membres de l'église française de Threadneedle) et finalement du philanthrope Thomas Firmin ${ }^{19}$. Assistaient en outre aux réunions deux Anciens de Threadneedle, Guillaume Carbonnel et Claude de Hayes.

Dans ces circonstances, les huguenots-Pierre Jurieu et Élie Benoist en tête-firent naturellement de Charles II le héros de l'exil de $1681^{20}$. Le 19 octobre, une harangue fut prononcée devant le monarque par le pasteur André Lombard en signe de remerciement, harangue rapidement traduite en anglais, publiée à Londres et à Dublin, et promptement envoyée en France où Élie Benoist nous dit qu'elle fit 'beaucoup de bruit'. ${ }^{21}$ Lombard admet 'peu s'en faut que je ne m'Emporte jusq'aux derniers Excés de l'Eloquence', tant ses remerciements sont enflammés ${ }^{22}$.

Charles, héros de l'exil huguenot? Et pourtant, Lombard, ministre de l'église de la Savoie, avait consenti à adopter l'anglicanisme. Il faut donc tout d'abord séparer son intervention de celle du ministre de la plus ancienne église française, Threadneedle, qui était dans une position toute différente du fait de son 'non-conformisme'. Threadneedle dépêcha donc David Primerose dont les remerciements, plus courts et plus sobres que ceux de Lombard, furent aussi imprimés (mais en deux colonnes, l'une en français, l'autre en anglais), Remerciement fait au roi de la part de l'Eglise Francoise et de l'Eglise Flamande de la ville de Londres (Londres, 1681). Outre cette différence entre les églises françaises qui témoigne de leurs difficultés à communiquer même dans les pires circonstances, que lit-on entre les lignes, par exemple d'un Jurieu? Que les déclarations de Charles II étaient aussi 'capables de le justifier des injustes soupçons qu'on a eus contre luy à l'égard de sa Religion ${ }^{23}$. Dans un même esprit, le comte de Halifax, le frère de l'envoyé extraordinaire de Charles à Paris, Henry Savile, lui avait écrit qu'à l'été 1681, en Angleterre, faire bonne figure aux réfugiés français pouvait être très utile pour quiconque était soupçonné de papisme: 'In particular I shall endeavour to justify my

\footnotetext{
${ }^{19}$ The Currant Intelligence, $\mathrm{n}^{\circ} 42$ (du 13 au 17 septembre1681).

${ }^{20}$ Benoist, t. IV, p. 491. Jurieu décrit et traduit les lettres de juillet dans Les Derniers Efforts, I, pp. 40-47. Il semble croire que la naturalisation a été accordée aux Protestants français, ce qui n'était pas en fait le cas (p. 47). Voir également sa description du Popish Plot, entamée dans La Politique du Clergé de France, pp. 130-146 et poursuivie dans Les Derniers Efforts, II, pp. 96-130. Pour les remerciements des églises françaises, voir Luttrell, vol. 1, p. 138 ; Souloudi et Hands, pp. 7-9 ; Minutes of the Consistory of the French Church of London, Threadneedle Street, 16791692, ed. Robin Gwynn, Huguenot Society of Great-Britain and Ireland, Quarto Series LVIII (Londres, 1994), p. 57.

${ }^{21}$ Benoist, t. IV, p. 491. Voir [André Lombard], Harangue au Roy: Faite par un Ministre de l'Eglise Francoise de la Savoye (Londres, 1681); A Harangue to the King. By a Minister of the French Church in the Savoy, the Nineteenth Of October, 1681. In the French Tongue (Londres, 1681) et $A$ Speech to the King : Made by a Minister of the French Church of the Savoy, The 19th, of October, 1681. In the French Tongue (Dublin, 1681). Les versions publiées en Angleterre et en Irlande ne sont pas du même traducteur.

22 [Lombard], Harangue, p. 7.

23 Jurieu, Derniers Efforts, I, p. 41.
} 
Protestantship by doing all that is in my power towards the encouragement of those that shall take sanctuary here out of France, 24 .

Alors qu'ils tentaient de se remettre des tentatives d'exclusion du duc d'York lors des derniers parlements, Charles et son clergé avaient donc tout intérêt à apparaître comme défenseurs du protestantisme européen, à 'justifier' leur protestantisme, au risque d'offenser Louis XIV. Charles, qui a nul doute longuement pesé les conséquences de son geste, a peut-être été influencé par le déchaînement des passions de ses adversaires whigs qui lui reprochaient déjà sa politique de rapprochement avec la France. Pendant que le roi réfléchissait aux conditions d'accueil des exilés, le non-conformiste Richard Janeway (connu dans les milieux de l'édition pour ses activités séditieuses) fit imprimer The Deplorable State and Condition of the Poor French Protestants Commiserated ... With Reasons for a Protestant League, un petit texte d'une dizaine de pages peu connu des chercheurs, mais l'un des plus ardents manifestes en faveur du droit d'ingérence. Janeway (qui est peut-être lui-même l'auteur du texte) en appelle sans détour à une union des princes protestants, inspirée par les lois de Dieu, de la nature, et des nations, contre des persécutions qu'il compare au Massacre des Innocents et à la Saint Barthélemy ${ }^{25}$. L'appel à Charles est à peine voilé : 'deserters of their Brethren in their time of Danger and Distress, are more obnoxious and guilty, than the Tyrants themselves that Persecute them ${ }^{26}$. Le peuple protestant français doit être libéré du joug d'un tyran idolâtre. En n'intervenant pas, Charles risque, au mieux, d'entrer lui-même dans l'histoire comme un tyran, au pire, d'encourir les foudres divines. La relation entre catholicisme et pouvoir absolu était sur toutes les lèvres en Angleterre et les récits de dragonnade entraient ainsi d'emblée dans la rhétorique anti-absolutiste whig ${ }^{27}$.

De telles publications ont dû hâter la décision du roi, qui était en parallèle tenu au courant de la situation par son envoyé, Henry Savile. Le protestantisme de Savile n'était plus à prouver ; il fréquentait régulièrement le temple de Charenton quand d'autres anglicans s'en détournaient sous prétexte que les ministres français n'étaient pas ordonnés par le pouvoir épiscopal. Il avait à maintes reprises suggéré à Charles des gestes symboliques et pratiques de plus grande ampleur que ceux qui furent finalement consentis ${ }^{28}$. Par exemple, persuadé qu'un coup d'éclat était nécessaire, Savile conseilla à son roi de se déclarer ouvertement défenseur du protestantisme en publiant simultanément en latin et en français une condamnation des violences faites aux

\footnotetext{
${ }^{24}$ Savile Correspondence, p. 212 (28 juillet-7 août 1681). C'est nous qui soulignons.

${ }^{25}$ The Deplorable State and Condition of the Poor French Protestants Commiserated... With Reasons for a Protestant League (Londres, 1681), pp.1, 10.

${ }^{26}$ Ibid., p. 6.

27 Voir par exemple, Melinda S. Zook, Radical Whigs and Conspiratorial Politics in Late Stuart England (Pennsylvania State UP, 1999), xvii.

${ }^{28}$ Savile Correspondence, pp. 189, 193, 197-198, 201, 212-214
} 
huguenots et en ordonnant la naturalisation de tous les réfugiés, une mesure que l'ambassadeur appelait de ses vœux depuis longtemps, mais qui était devenue irréalisable en l'absence de parlement :

Now should his Maj[es]tys circumstances admitt of these measures, were not the propperest method to begin with a declaration to all Europe in Frenche and Latin to offer countenance and encouragement to all such as receaving prejudice from the profession of the Prot. Rel. in any other countryes could come and harbour themselves in his? [...] whilst the present misrepresentations given by some ill men of the present posture of our affaires make people ballance, who would flye to England upon the first publick assurance they should bee well receaved there.... but all this was upon the hopes of a bill of naturalization, which soe unfortunately fayling lessen'd my credit with them as well as my hopes of doeing a considerable service to the nation ${ }^{29}$.

La naturalisation, assortie de sauf-conduits autorisant les artisans à voyager avec leurs outils, devait permettre à l'Angleterre d'attirer de 'riches' exilés qui, sans cette garantie, préféreraient émigrer en Hollande. Savile, en bon diplomate, savait tempérer son émoi par des arguments économiques imparables, mais il se sentit désavoué lorsque la naturalisation devint clairement impossible (elle ne sera finalement accordée qu'en 1709) ${ }^{30}$.

Charles ne consentit donc finalement qu'à une seule des mesures proposées par son ambassadeur (laisser les artisans voyager avec leurs outils) et ne se permit certainement pas de donner son opinion à Louis, comme Jenkins semblait le lui suggérer. Par contre, il tenta à plusieurs reprises de faire pression sur le consistoire de l'église de Threadneedle qui, contrairement à celle de la Savoie, n'avait pas embrassé la liturgie et la discipline anglicanes. Les aides financières étaient subordonnées à l'obéissance civile et religieuse des huguenots, ce qui signifiait pour le monarque étouffer toute tentative de soutien aux presbytériens calvinistes anglais ou au parti Whig. Par exemple, le 30 octobre 1681, Guillaume Carbonnel ayant accepté de servir dans le jury qui avait acquitté Sir John Rouse (un Whig accusé de trahison), le roi fit connaître sa colère au consistoire, d'autant plus que Carbonnel participait aux réunions du comité chargé de la répartition des aides aux réfugiés. Les termes trahissent ce qu'il faut bien appeler le chantage financier de Charles : 'the King was indignant about this Carbonnel matter and that he would have to warn the foreigners here that in future they should not meddle in any public business or the King would no longer favour them with his protection, ${ }^{31}$. Le lendemain, une lettre de Sir John Chardin fait part des inquiétudes des autorités : 'It is essential for the poor

\footnotetext{
${ }^{29}$ Ibid., p. 209-210.

${ }^{30}$ Ibid., p. 236.

${ }^{31}$ Minutes of the Consistory, p. 65.
} 
Protestants that nothing is done which might make the King take a dislike to them and give colour to their enemies' claims that they are Presbyterians and Republicans, ${ }^{32}$.

En juillet 1681, au moment où les rumeurs de dragonnade commencèrent à atteindre les lecteurs anglais, Charles II se décida donc à prendre la défense des huguenots, mais poussé par ses adversaires politiques, par la pression internationale et sans doute aussi par son clergé, soucieux de représenter le chef de l'église anglicane comme un bon protestant sur la scène internationale $^{33}$. Le roi semblait avoir hésité entre sa compassion pour des frères persécutés (et la possibilité de retombées économiques) et la peur de faire pénétrer en son royaume des hordes de dissidents républicains. Ces mêmes hésitations entre charité chrétienne et peur de l'inconnu se retouvent dans la majorité des récits des événements de 1681 .

\section{Exilés et traîtres}

Avant même que Charles II ne se soit prononcé en faveur des exilés, les auteurs anglais sont entrés en compétition pour informer leur lectorat soit de la mauvaise foi des huguenots, soit, au contraire, de l'ampleur de leur souffrance et de l'accueil qui devait par conséquent leur être réservé. Par exemple, lorsque Richard Janeway adresse à Charles ses pressants encouragements pour une union protestante, il se heurte à une objection toute simple : et si les huguenots étaient des traîtres, leur exil le cheval de Troie du Pape ? Janeway tente de démontrer l'absurdité d'un tel propos : il faut d'abord regarder les conditions de l'exil. Les huguenots arrivent sur des bateaux si petits et si fragiles qu'ils risquent leur vie à chaque instant. Seuls des êtres désespérés entreprennent un tel voyage. Ensuite, ce ne sont pas seulement des hommes qui arrivent en Angleterre, mais des femmes et des enfants; ce ne sont pas des soldats en armes, mais des pêcheurs et des artisans du textile. Ils ont, de plus, déjà été acceptés dans d'autres pays, notamment en Hollande. Enfin, comment des 'milliers' de fugitifs qui arrivent 'de toutes parts' pourraient-il coordonner une insurrection ? Janeway conclut que les papistes n'ont vraiment rien à gagner de l'exil huguenot qui leur donne mauvaise presse dans l'Europe toute entière ${ }^{34}$.

Les points de vue divergents sur les huguenots peuvent être examinés à partir de deux exemples précis. Le premier est un recueil de lettres adressées par un gentleman londonien à un ami 'à la campagne' intitulé The Present State of the Protestants in France. In Three Letters. Il connut un certain succès puisqu'il reparut deux ans plus tard dans une version augmentée sous le titre, An Apology for the Protestants of France, in Reference to the Persecutions they are under at this day; in Six Letters (Londres, 1683, parfois attribué à tort à Roger L’Estrange).

\footnotetext{
${ }^{32}$ Ibid., p. 67.

${ }^{33}$ Voir les conclusions de Robin Gwynn sur le sujet, Huguenot Heritage, pp. 162-163, 167.

${ }^{34}$ Janeway, Deplorable State and Condition, p. 10.
} 
Nous avons ici un texte 'mixte' qui certes se présente sous la forme d'un récit épistolaire, mais l'échange de lettres entre le londonien et le provincial est en fait subordonné au dialogue entre le londonien et un ami qui vient de rentrer de Paris. Cet ami a ramené de son voyage en France une caisse entière de livres et de feuilles volantes (les édits et les déclarations de Louis XIV) que le narrateur est libre de consulter à sa guise pour assouvir sa propre soif d'information et pour pouvoir ensuite en faire part au provincial qui n'a pas d'accès direct aux dernières nouvelles de la capitale ${ }^{35}$.

Alors qu'il se félicite des mesures prises par Charles II, le londonien ne peut toutefois s'empêcher d'avouer qu'il a entendu dire que les persécutions ne seraient pas aussi violentes qu'on le dit :

It is confes'd, that their King is very earnest to make them embrace his Religion: but they assure us, that he uses none but very Reasonable Means, and that they who come hither with such Outcries, are a sort of People not gifted with much patience, who easily forsake their Native Country, being dissatisfied, that their merit, as they conceive, is not sufficiently rewarded. Besides, they are represented to us very much suspected in the point of their Obedience and Loyalty ${ }^{36}$.

Son ami l'interrompt en attribuant ces mensonges au Père La Chaise qui tente par tous les moyens d'empêcher que les voisins de la France ne fassent bon accueil aux protestants en sousestimant l'ampleur des persécutions. Par conséquent, la première lettre est tout entière consacrée à une présentation de l'Édit de Nantes, parce que ce rappel permet de s'attaquer aux mensonges des catholiques, comme aux idées préconçues des Anglais sur les réfugiés. La méfiance religieuse et économique à l'égard des huguenots était telle qu'ils passaient en effet soit pour des catholiques déguisés, soit pour des profiteurs ${ }^{37}$. À l'inverse, pour le narrateur, les huguenots doivent être accueillis à bras ouverts parce que c'est sur eux que se vengent les catholiques déçus de ne pas avoir pu renverser la monarchie anglaise lors du complot de 1678 (Popish $P l o t)^{38}$. La seconde lettre, un 'récital de calamités' pour reprendre les termes même de l'auteur, passe en revue les différents textes, édits et déclarations à l'encontre des protestants ${ }^{39}$. Les explications restent encore très pédagogiques : après des considérations sur l'Édit de Nantes, on assiste maintenant à des éclaircissements sur ce que signifie, par exemple, 'faire amende honorable ${ }^{40}$. Il paraît clair que l'auteur suppose que ses lecteurs ignorent, dans l'ensemble, la réalité de la répression légale.

\footnotetext{
${ }^{35}$ State of the Protestants, p. 3.

${ }^{36}$ Ibid., p. 4

${ }^{37}$ Ibid., sig. a1rv.

${ }^{38}$ Ibid., sig. A2v, p. 11.

${ }^{39}$ Ibid., p. 20.

${ }^{40}$ Ibid., p. 13.
} 
L'auteur prend ici un soin tout particulier à opposer la dragonnade à la St Barthélemy, ce qui peut sembler curieux ${ }^{41}$. Selon lui, les conséquences sont les mêmes, mais les moyens employés différent radicalement. Il se sert d'un effet d'accumulation de publications officielles pour montrer que l'on tue les huguenots à petit feu, mais se garde bien d'entrer de prime abord dans les détails des tortures physiques qui pourraient passer pour mensongères. En mentionnant que les édits et autres déclarations non seulement portent atteinte à la liberté de culte, mais aussi assassinent les huguenots en les privant de leurs moyens de subsistance, il peut prouver ses dires 'officiellement' sans avoir recours aux récits les plus crus : en France, le huguenot ne meurt pas parce que les dragons lui brûlent les pieds, lui font avaler des gallons d'eau ou le pendent au plafond, mais parce qu'il ne peut pas moissonner ou faire commerce.

Notre deuxième exemple, qui présente un point de vue radicalement différent, est une traduction anonyme des actes de l'assemblée du clergé, Actes of the General Assembly of the Clergy of France (Londres, 1682), ou plus précisément de 1'AAvertissement Pastoral' aux membres de la R.P.R. et ses 'méthodes' pour leur conversion ${ }^{42}$. Ce texte est accompagné d'une préface d'une violence remarquable à l'égard des huguenots dont le sort est comparé à celui des catholiques persécutés lors du complot papiste prétendument déjoué en Angleterre en 1678. Dès les premières lignes, on sent poindre l'imitation ironique des récits de violence: 'happy is he, that can escape into England with burning Match between his Fingers or red hot Iron Plates fixed to the Soals of his Feet ${ }^{43}$. Alors que l'auteur de la préface se dit animé d'une charité toute chrétienne envers ceux qui sont persécutés pour leur religion, il refuse de croire que ce peut être le cas des huguenots ; ces 'vagabonds', issus des 'égouts' de la France qui mangent le pain des Anglais, sont des espions à la solde de Rome et ne s'exilent que par fainéantise :

I am loath our Nation should be imposed upon, by Shams, and Cheats, on Religious pretexts ; I am loath, I say, the Town and Country should be incumbred ; our own Poor deprived of Alms, the Bread eaten out of honest Tradesmen mouths; and we made, as it were, the Sink of France by countenancing, and entertaining from thence, swarms of Vagabonds, amongst whom some are Papists in Masquerade, and others at best, such Protestants as hate Episcopacy, worse

\footnotetext{
${ }^{41}$ Ibid., p. 25.

${ }^{42}$ Le ton violent de la préface fait conclure Schickler que son auteur est sans doute un catholique français, un point qui n'est guère corroboré par les inquiétudes du traducteur concernant les relations possibles entre les huguenots et les catholiques ; voir Fernand de Schickler, Les Eglises du Refuge en Angleterre, 3 vols (Paris, 1892), vol. 2, p. 308. Il est bien plus probable que nous ayons ici affaire à un anglican tory et High Church, prêt à reconnaître que les catholiques représentent une menace moindre pour le gouvernement que les dissidents protestants. Pour des considérations sur l'avertissement, voir Pierre Blet, Les Assemblées du Clergé et Louis XIV de 1670 à 1693 (Rome, 1972), pp. 433-37 et Jean-Robert Armogathe, Croire en la liberté. L'Église catholique et la révocation de l'Édit de Nantes (Paris, 1985), pp. 55-104. Pour les réponses des huguenots, voir Jean Claude, Considérations sur les Lettres Circulaires, de L'Assemblée du Clergé de France (La Haye, 1683) et Claude Pajon, Remarques sur l'Avertissement Pastoral (Amsterdam, 1685).

${ }^{43}$ Actes of the General Assembly of the Clergy of France (Londres, 1682), sig. A2r.
} 
than Popery, and come hither upon no other Persecution then what Idleness, beggery, or some misdemeanor hath laid upon them ${ }^{44}$.

On retrouve ici une rhétorique commune à toutes les époques, qui mêle une xénophobie latente à des arguments pseudo-économiques. L'immigration huguenote a toutefois cette particularité : elle a pour origine un mensonge, une tromperie sur l'état du protestantisme français que les huguenots propagent soit par profit, soit parce qu'ils sont envoyés par Rome. L'année suivante, le Whig Gilbert Burnet (récompensé par un évêché après la Glorieuse Révolution) proposa sa propre version sous le titre The Letter Writ by the Last Assembly General of the Clergy of France to the Protestants (Londres, 1683) pour s'attaquer à la précédente et plaider en faveur des huguenots ${ }^{45}$.

Malgré l'aide de Burnet (et de beaucoup d'autres), les attaques portées contre les huguenots au moment de leur exil en 1681 s'attachaient à raviver une peur quasi ancestrale chez les anglais : celle que la Manche, qui avait fourni à plusieurs reprises une protection efficace contre les invasions catholiques, allait, cet été là, déverser des ennemis du royaume dans tous les ports d'Angleterre. Henry Savile savait ce qui se passait en France, Charles II était au courant des exactions du Poitou, des nobles revenaient de France en ayant vu de leurs propres yeux des huguenots passés au feu. Il est moins certain que le boutiquier de Douvres ait été persuadé que les réfugiés qui débarquaient au port en parlant la langue des papistes aient été aussi à plaindre qu'ils le prétendaient. Le thème du 'papiste masqué' ('Papist in Masquerade') que l'on retrouve dans la littérature de controverse à la fois whig et tory de la Crise de l'Exclusion nous rappelle la complexité des représentations : les Tories pouvaient être accusés de papisme étant donné leur attachement aux cérémonies de l'église anglicane ; mais également les Whigs (et donc leurs alliés non-conformistes et huguenots) car ils tentaient d'affaiblir la religion du royaume et jouaient ainsi le jeu de Rome ${ }^{46}$.

Avec les huguenots, l'accusation de papisme prend un tour particulier. Un détail qui n'a pas vraiment retenu l'attention des historiens a peut-être contribué à de telles accusations : l'impossibilité de communiquer avec les exilés. On pouvait 'voir' les souffrances sur les corps mais pas toujours entendre le récit des persécutions de la bouche de ceux qui en portaient encore les traces dans leur chair - et ce malgré l'utilisation du français comme lingua franca de l'élite européenne et la présence sur les bateaux de réfugiés pouvant servir de traducteurs. Dans ce

\footnotetext{
${ }^{44}$ Ibid., sig. A2r.

${ }^{45}$ Burnet n'était pas un novice de la question française puisqu'il avait déjà composé un récit sur la St Barthélemy intitule A Relation of the Barbarous and Bloody Massacre of About an Hundred Thousand Protestants (Londres, 1678) et Strange News from France: In a Letter giving a Relation of the Present State of the Difference Between the French King and the Court of Rome (Londres, 1682).

${ }^{46}$ Voir Tim Harris, London Crowds in the Reign of Charles II. Propaganda and Politics from the Restoration until the Exclusion Crisis (Cambridge, 1987), pp. 139-140, 153 et Lois G. Schwoerer, The Ingenious Mr. Henry Care, Restoration Publicist (Baltimore et Londres, 2001), pp. 157-166.
} 
contexte, il faut citer une autre publication de Janeway : le compte-rendu de l'arrivée de vingt protestants de Bordeaux, inséré dans le journal (dont certains numéros furent composés par Henry Care et Thomas Vile) que Janeway éditait à l'époque, The True Impartiall Mercury. Voici un extrait du mois d'octobre dans lequel Janeway explicite certaines méthodes de torture, selon lui peu connues du lectorat anglais :

This Day put into the Port about Twenty Distressed French Protestants In a Ship from Burdeaux. To which place they fly in great Number to get Shipping for England, being most extreamly Persecuted, some of that number having the Soles of their Feet burnt with flaming Brass, to force them to Abjure their Religion, And one of this Company had been, Wollen'd (as they Phrase it) which is to have a Cord put around their Temples, and then Twisted with a stick behind, which almost forces their Eyes out of their heads, nay their cruelty Reaches so far as that they force sometimes even the Children to put one of their Fingers into a small hole, and then drive a wedge upon it, with several other Barbarities which seem almost Incredible, had we not seen and Examined these poor Soules just upon their Landing, not being then Cured of some of the Wounds occasioned by their Tortures ${ }^{47}$.

On retrouve ici précisément les descriptions de souffrances dont se moque plus tard le traducteur de 'L'Avertissement Pastoral'. Il est cependant intéressant d'observer la façon dont Janeway, en tant que 'journaliste', fait part de ses commentaires, qui sont souvent très explicites. Il 'observe' et 'examine' les corps des huguenots qui portent les traces (on n'ose dire les stigmates) de leurs récentes souffrances, mais à qui Janeway ne donne jamais la parole directement. Il 'voit' en quelque sorte les tortures, plus qu'il ne les entend, malgré la présence indéniable de passagers qui ont pu lui expliquer les techniques de torture et le nom des instruments utilisés ('as they Phrase it'). La parole huguenote, nous y reviendrons, ne se fait entendre que par l'intermédiaire de témoignages anglais.

On aurait donc fort tort de supposer que l'accueil des huguenots fut unanimement chaleureux. Mais plus que des accusations générales (et en ce sens peut-être prévisibles) de profit économique, nous sommes ici confrontés à la résurgence de peurs profondes liées au manque de connaissance des protestants français : peur de leurs penchants anti-monarchistes, mais également peur de leurs liens avec Rome. En Angleterre, les deux étaient d'ailleurs liées puisque le complot de 1678 - qui devait assassiner Charles avant de rétablir le catholicismeétait encore frais dans les esprits et que les pamphlétaires utilisaient sans réserve la rhétorique du 'papiste masqué' pour quiconque n'était pas de leur avis. Autrement dit, ce que l'on reproche aux huguenots (comme on le reproche aux Tories, aux Whigs, aux anglicans et aux dissidents) c'est tout simplement de n'être pas protestants. Ces rumeurs pouvaient avoir d'autant plus prise sur l'opinion publique que les huguenots s'exprimaient dans une langue papiste: le français a

\footnotetext{
${ }^{47}$ The True Impartiall Mercury, ${ }^{\circ} 48$ (du 4 au 7 octobre 1681).
} 
maintenant remplacé l'espagnol. Déloyaux, monarchomaques, républicains (on le savait déjà), les huguenots sont aussi traîtres, menteurs, profiteurs : ils n'hésitent pas à abuser de la charité chrétienne et de la compassion de leurs frères pour servir leurs propres intérêts ou celui de leur maître, Innocent XI.

Nous verrons dans la dernière partie de cet article comment ces allégations (qui sont souvent anonymes) ont été attribuées par les Whigs aux Tories, qui étaient eux-mêmes accusés de collusion avec Rome. En 1681, cependant, la grande frayeur catholique laissait peu à peu la place dans les esprits à la peur d'une alliance entre les dissidents radicaux et les Whigs contre la monarchie et l'anglicanisme. A cette peur du huguenot catholique vint donc s'ajouter la peur du huguenot dissident, qui, cette fois, peut être directement attribuée aux Tories. Les huguenots, en effet, pouvaient faire basculer le fragile équilibre des confessions en Angleterre en se déclarant ouvertement du côté des non-conformistes anglais, des presbytériens en particulier. Les Tories se trouvaient donc face à deux stratégies, dont ils usèrent d'ailleurs simultanément : accuser les huguenots de dissidence et tenter de rallier les ministres français les plus respectés à l'anglicanisme.

Écrire l'exil : la variété des discours

Les répliques aux violentes diatribes contre les huguenots furent nombreuses mais conditionnées, en quelque sorte, par les attaques. À l'été 1681, la publication des lettres de Charles II, la collecte organisée dans la capitale (dont les conditions devaient être lues en chaire dans toutes les églises paroissiales), les récits, souvent courts et donc relativement abordables pour un grand nombre de lecteurs, le rappel de la législation contre les huguenots, avaient une fonction pédagogique essentielle : transformer le huguenot de suspect en victime. Pour ceux qui ne doutaient pas de la loyauté et du protestantisme des huguenots, ce rassemblement de surface masquait toutefois de profondes divisions sur la meilleure façon de se 'servir' de ce nouvel afflux de protestants.

Avant même de commencer à analyser ces textes, il nous faut remarquer que si la dragonnade fut sévèrement condamnée à l'étranger, les bonnes intentions de certains contribuèrent à la détérioration des relations entre protestants et catholiques en France. Dans un dialogue de 1683 inspiré des Derniers Efforts de l'Innocence Affligée que Paul Fétizon fit imprimer à La Haye, Patrice, le catholique, reproche à son ami Eusèbe, le huguenot, de s'en prendre dans de 'mechans livres, qui sont plûtost des libelles diffamatoires', non seulement aux 
persécuteurs, mais à Louis XIV lui-même ${ }^{48}$. La réponse d'Eusèbe est éloquente. Premièrement, il doute que toutes les publications de ce type en langue française soient effectivement issues de la plume de huguenots, le français étant devenu la langue européenne de diffusion des connaissances $^{49}$. Deuxièmement, Eusèbe reconnaît que certains étrangers ont poussé la charité chrétienne un peu trop loin en dénonçant le roi. Ces récits portent la 'marque' de leurs origines, la marque de patries où chacun est 'libre' de s'exprimer (et en ce sens on ne saurait les censurer), mais ils ne doivent en aucun cas être imputés aux protestants français qui ne cherchent qu'à prouver au monarque leur éternelle loyauté : 'lors que nous voyons des gens, que la compassion de nos maux emporte au delà des bornes, nous leur dirions volontiers, ou ne vous mêlez pas de nos affaires, ou rendez vous capables de nos sentimens, ${ }^{50}$. Eusèbe semble à la fois s'enorgueillir de l'élan de charité de ses frères protestants et le déplorer, comme il déplore de se donner en spectacle, dans la citation en exergue de cet article: 'Nous sommes devenus le spectacle de la terre par nos calamitez ${ }^{51}$.

Il s'agit donc bien de la production d'un 'spectacle', de la théâtralisation de la souffrance, auxquelles non seulement les huguenots, mais aussi les commentateurs extérieurs furent confrontés. Comment, de l'étranger, dénoncer avec conviction les persécutions, tout en ne contribuant pas à envenimer la situation ? N'avait-on pas souvent tendance à l'exagération pour tirer profit de la souffrance des huguenots? Les réponses varièrent évidemment en fonction de la situation des observateurs. Comme l'a très justement montré Hans Bots, les gazetiers de Hollande, par exemple, se contentèrent de rapporter les faits le plus objectivement possible, sans tomber dans le récit des tortures, et ce, de peur de voir la situation des huguenots restés en France empirer ${ }^{52}$. Les Anglais n'étaient pas, bien sûr, insensibles à l'argument, comme le fait remarquer Halifax : 'there is great tenderness to be used in the manner of it, that we may give no occasion for a higher persecution against them there, or by disputing a prince's power over his own subjects, ${ }^{53}$. Non seulement Halifax recommande la prudence, mais, contrairement à Janeway, il doute du bien-fondé de l'ingérence dans les affaires de Louis XIV, un argument qui ressurgira lorsqu'il sera question pour Jacques II de condamner la révocation de l'Édit de Nantes quatre ans plus tard. Malgré ces appels à la prudence qui émanent des cercles aristocratiques et diplomatiques, les Anglais, pris dans la tourmente de la politique intérieure, ne

\footnotetext{
${ }^{48}$ [Paul Fétizon], Apologie pour les Reformez, Où On voit la juste Idée des Guerres Civiles de France : Et Les vrais Fondements de l'Edit de Nantes (La Haye, 1683), p. 154.

${ }^{49}$ Ibid., p. 216.

${ }^{50}$ Ibid., p. 215

${ }^{51}$ Ibid., p. 215.

${ }^{52}$ Hans Bots, 'L'écho de la Révocation dans les Provinces-Unies à travers les gazettes et les pamphlets', dans Zuber et Theis, La Révocation, pp. 281-298. Pour l'émigration des réfugiés en Hollande, voir Solé, 'La diplomatie de Louis XIV'.

${ }^{53}$ Savile Correspondence, p. 212.
} 
sauront pas, ou ne pourront pas, adopter le même ton de modération que leurs confrères néerlandais, en particulier dans la presse populaire.

Il nous faut d'abord souligner l'extraordinaire variété des publications anglaises qui parurent dans les quelques mois précédant et suivant la dragonnade du Poitou. Elles s'organisent autour de quatre thèmes principaux : le compte-rendu des déboires du clergé gallican avec Rome, les reproductions des édits et des déclarations, les tentatives huguenotes pour mettre fin aux persécutions, et les récits de la dragonnade ${ }^{54}$. Ce sont ces récits que nous nous proposons ici d'étudier, ainsi que la variété des publications dans lesquelles ils parurent: les feuilles imprimées (broadsides), les opuscules, les échanges épistolaires et la 'presse d'information, ${ }^{55}$.

Les récits de dragonnade se rencontrent en règle générale dans des textes courts qui tentent un résumé des événements : la responsabilité de Marillac, les violences sur les biens et les personnes, en particulier les enfants et les personnes âgées, et la loyauté monarchique des huguenots. La plupart de ces textes choisissent cependant un angle d'approche précis : une déclaration ou une anecdote particulières. On peut citer par exemple The Horrid Persecution of the French Protestants in the Provinces of Poitou (Londres, 1681) qui prend la forme d'une lettre d'un Anglais à un ami de Cantorbéry. Cette feuille imprimée se veut en partie un commentaire de la déclaration du 17 juin (mise en pratique le 18 juillet) qui abaisse l'âge du consentement pour les conversions à sept ans et oblige, en théorie, les parents huguenots à payer la pension d'un enfant qui émettrait le souhait de recevoir une instruction catholique ${ }^{56}$. C'est ainsi que les dragons pénétraient dans les maisons, ces 'petits azyles inviolables, ${ }^{57}$, mais aussi que les catholiques avaient accès à l'âme des plus faibles, privant du même coup le chef de famille huguenot de l'autorité qui garantissait le maintien du tissu social : on peut changer sa religion à un âge où seules comptent 'une pomme \& une piroüette' ${ }^{58}$. Henri Savile s'était bien rendu compte de la portée psychologique de la déclaration : 'The edict I mentioned in one of my last concerning the Huguenots and theire children dos soe alarum them that they are making

\footnotetext{
${ }^{54}$ Pour l'entrevue de la dernière chance entre le marquis de Ruvigny et Louis XIV, voir Solange Deyon, Du Loyalisme au refus. Les Protestants français et leur député général entre la Fronde et la Révocation (Lille, 1976), pp. 115-125, 131-147 et Garrisson, pp. 166-168.

${ }^{55}$ Voir également les traductions de grandes figures du refuge comme Jurieu. La Politique du Clergé de France fut traduit à Londres dès 1681 sous le titre The Policy of the Clergy of France et Les Derniers Efforts de l'Innocence Affligée en 1682, The Last Efforts of Afflicted Innocence (1682). Pour de longues citations et un commentaire de Jurieu, voir aussi An Apology for the Protestants of France, in Reference to the Persecutions they are under at this day; in Six Letters (Londres, 1683), sixth letter, pp. 74-94.

${ }_{56}$ Pour la déclaration, voir Pilatte, pp. 88-90.

${ }^{57}$ Une expression de Jurieu, Derniers efforts, I, p. 62.

${ }^{58}$ Ibid., I, p. 75. Voir également The True Protestant Mercury (Janeway), $\mathrm{n}^{\circ} 28$ (du 26 au 29 juillet 1681) et The True Protestant Mercury de Langley Curtis, $\mathrm{n}^{\circ} 58$ (du 23 au 27 juillet 1681). Pour les derniers instants des huguenots, voir les déclarations du 19 novembre 1680, du 7 avril 1681 et du 20 juin 1681, Pilatte, pp. 68-69, 75-77 et 91-92, respectivement.
} 
extraordinary deputations to the King to prevent it ${ }^{59}$. Le texte fut d'ailleurs rapidement traduit en anglais, dans plusieurs versions, comme The French King's Declaration, that the Children of those of the Pretended Reformed Religion may change their Religion at the Age of Seven (Londres, 1681) et Animadversions upon the French King's Declaration against the Protestants (Londres, 1681) qui commentent la déclaration. Voici certaines des conséquences qui y sont mentionnées :

And for fear it should be imagined, that I will here use Exaggeration, I beseech all those, who shall read these Considerations, to observe, to how many Mischiefs this Declaration will open the Gate. The Reformed will no longer be Masters in their own Houses: The Priests, the Monks, the Missionaries, the Bigots of both Sexes will enter into them, when they please, nor shall one dare refuse to open them the door; they will take the Children aside in the presence of their Parents, they will have the leisure to cajol them as long as they please, none daring to contradict, or whisper the least word against it, upon pain of being treated as Rebels and Criminals ${ }^{60}$.

Lorsqu'Élie Benoist félicite Charles II pour avoir été le grand pionnier de la défense du protestantisme, c'est aux conditions d'accueil des enfants qu'il fait précisément référence ${ }^{61}$.

A Strange but true Account of the Barbarous usage of three young Ladies in France for being Protestants (Londres, 1681), prend lui aussi pour point de départ un fait particulier, mais dans un tout autre registre. Il s'agit de l'histoire de trois belles orphelines normandes, emprisonnées par un jésuite après le mariage de l'une d'entre elles avec un catholique de quelques dizaines d'années son aîné. Les demoiselles sont finalement secourues par un gentleman huguenot qui organise leur fuite en Angleterre après une évasion spectaculaire de la fenêtre du donjon. Pour tous ceux qui seraient tentés de douter de la véracité du récit, le texte précise que les lettres 'originales' peuvent être librement consultées dans une coffee house de Covent Garden. Les événements de France sont ici narrés dans un récit dont le titre accrocheur était probablement crié pour appâter le chaland. C'est le seul texte de cette nature que nous ayons pu retrouver, mais qui nous rappelle que les événements de France n'étaient pas seulement traités sur le mode de l'information 'objective' et pouvaient aussi être envisagés comme une source de profit facile par les auteurs de la littérature de grande distribution. D'ailleurs, le titre de certains textes rappelle celui des ballades ou canards colportés comme News from France (1682) de Gilbert Burnet ou Strange and Wonderful News from France (1682), qui tous deux traitent de la possible indépendance de l'église gallicane.

\footnotetext{
${ }^{59}$ Savile Correspondence, p. 201. Savile mentionne à nouveau dix jours plus tard que les huguenots sont davantage sensibles à cette mesure qu'à toutes celles qui l'ont précédée (p. 206, lettre du 12 juillet).

${ }^{60}$ Animadversions, pp. 17-18.

${ }^{61}$ Benoist, t. IV, p. 491-492. Pour les conséquences de la déclaration en France voir t. IV, pp. 445-459. Pour Charles comme défenseur des enfants protestants, voir aussi [Lombard], Harangue, p. 5.
} 
Certains textes peuvent ensuite être directement liés aux milieux whigs ou non-conformistes et c'est en règle générale dans ces publications que l'on trouve les récits les plus sensationnels, un véritable 'spectacle des calamitez'. Nous avons déjà rencontré l'exemple de Janeway et son récit de torture des Bordelais. En janvier 1682, il publia A True and Perfect Relation of the New Invented Way of Persecuting the Protestants in France qui rappelle brièvement les déclarations et édits les plus significatifs depuis 1679, puis procède à un examen de la dragonnade mois par mois, d'avril à octobre 1681. On peut aussi citer An Abstract of the Present State of the Protestants in France (Londres, 1682) qui peint la dragonnade en des termes très crus et n'hésite pas à faire directement allusion au viol, apparemment une invention du compilateur anglais : '[The Dragoons] beat with cudgels the men, clap sword and pistols to their breasts; abuse and ravish the women: and when they have pillagd their houses, tie the Possessors to their horse tails, and drag them to Mass'.

Thomas Cockerill, un autre éditeur non-conformiste s'engagea, lui aussi, aux côtés des huguenots, mais en adoptant une technique toute différente. À l'inverse de son confrère Janeway, Cockerill n'a jamais publié d'opuscules à sensation sur la dragonnade, mais il est à l'origine de l'un des textes les plus importants de la période: le commentaire de 'E.E.' (Edmund Everard) sur les deux déclarations de Saint Germain, The Great Pressures and Grievances of the Protestants in France (Londres, 1681), dédié à Charles II ${ }^{62}$.

Fort de 82 pages, il s'agit du texte le plus long dans la catégorie des textes 'légaux' qui normalement s'attachaient à ne reproduire, en quelques pages, qu'un seul édit ou qu'une seule déclaration qui pouvaient ensuite être vendus pour quelques pennies. Même s'il ne s'agit pas véritablement d'une 'pétition' au monarque, le titre d'Everard et sa préface suggèrent que l'ouvrage fut conçu comme une adresse à Charles II pour exprimer les griefs des protestants français. En ce sens, il peut se comparer aux comptes-rendus qui circulaient en Angleterre des efforts des huguenots pour tenter de capter l'oreille de Louis XIV, The Humble Pettition of the Protestants of France to the French-King, To Recall His Declaration for taking their Children from them at the Ages of Seven Years (Londres, 1681) ou The Humble Petition of the Protestants of France, Lately presented to His Most Christian Majesty, by the Marechal Schomberg, and the Marquis of Ruvigny (Londres, 1681), ce dernier publié par un autre nonconformiste, Langley Curtis. À l'instar de Richard Janeway, Everard est un farouche défenseur du droit d'ingérence et pousse Charles II à intervenir auprès de Louis XIV. Pour ce faire, Everard reproduit d'abord la totalité de l'Édit de Nantes qui, selon lui, témoigne de l'intégrité et de la loyauté des huguenots. ${ }^{63}$ Il traduit ensuite, puis commente, chaque article des déclarations

\footnotetext{
${ }^{62}$ Everard était déjà connu pour son appel à une union de tous les princes protestants, Discourses on the Present State of the Protestant Princes of Europe (Londres, 1679).

${ }^{63}$ The Great Pressures, sig. A2v.
} 
de Saint Germain. Son texte était aussi, de toute évidence, destiné à accompagner les traductions des autres textes officiels. En effet, chaque fois qu'était publié un nouvel édit concernant les protestants français, le lecteur muni des traductions et surtout des commentaires d'Everard était capable de vérifier que la nouvelle législation ne contredisait pas les précédentes et, dans le cas contraire, d'en tirer les conclusions qui s'imposaient.

Il est peu probable que l'ouvrage ait été accueilli avec joie à la cour. D'abord, le juriste critique la tentative d'envoyer les huguenots dans les colonies américaines (Charles ayant encouragé leur exil en Caroline). Selon Everard, une telle pratique sert les intérêts du clergé gallican en affaiblissant le protestantisme européen ${ }^{64}$. Deuxièmement, il dresse un parallèle entre la situation des huguenots en France et celle des non-conformistes en Angleterre qui subissent les effets de sévères mesures de répression. Ainsi, lorsque Everard proclame que les membres du clergé 'break the Bonds of Charity and Christian Unity ... by encroaching upon the Civil Magistrates Power of making and executing of Penal Laws', il semble qu'il ait à l'esprit à la fois la situation de la France et celle de l'Angleterre, où sévissait le Code Clarendon contre les non-conformistes ${ }^{65}$. Everard en appelle finalement à la proclamation d'un 'Édit de Nantes' par Charles II pour garantir les libertés des protestants anglais, afin d'attribuer des 'privilèges limités' aux non-conformistes dont les principes ne sont contraires, ni à la morale, ni à la monarchie $^{66}$. Les autorités n'avaient pas grand intérêt, ne serait-ce que d'un point de vue purement pratique, à faire l'amalgame entre huguenots et non-conformistes comme le fait Everard. Par exemple, Thomas Earle, le maire de Bristol, désespéré par l'afflux de réfugiés indigents dans sa ville, proposa tout simplement au Secrétaire d'État d'utiliser les amendes levées contre les non-conformistes, pour aider financièrement les huguenots ${ }^{67}$.

L'objectivité et la rigueur des textes légaux sont contrebalancées par l'horreur des récits de torture, mais reste à observer une constante dans tous les textes de la période : aucun ne prétend être le récit direct d'un huguenot, si on admet une exception pour les deux textes de remerciements des églises françaises. Par exemple, nous avons observé plus haut le succès de l'échange épistolaire, The Present State of the Protestants in France. In three Letters (1681) et remarqué que l'ouvrage tend à ne pas divulguer des détails des souffrances physiques. Et pourtant, les cinq dernières pages du recueil opèrent un revirement soudain: en effet, le voyageur vient juste de recevoir des lettres d'‘amis' résidant encore à Paris qui lui présentent

\footnotetext{
${ }^{64}$ Ibid.

${ }^{65}$ Ibid. Pour une défense similaire des non-conformistes dont le sort est comparé à celui des huguenots, voir le sermon de Samuel Bold, le vicaire anglican de Shapwick dans le Dorset, A Sermon Against Persecution (Londres, 1683). Pour un commentaire, voir Mark Goldie, 'The Huguenot Experience and the Problem of Toleration in Restoration England', dans Caldicott et al., Huguenots in Ireland, pp. 184-188.

${ }^{66}$ The Great Pressures, sig. A2v.

${ }^{67}$ Voir les lettres des 10 décembre 1681 et 2 janvier 1681, citées par Ronald Mayo, 'The Bristol Huguenots, 16811791', PHS 21/5 (1970), pp. 443-444.
} 
cette fois la dragonnade dans toute son horreur physique: tortures, emprisonnements, maltraitances sur les enfants, les femmes et les vieillards ${ }^{68}$. Il faut cependant s'arrêter sur le statut de ces lettres et les techniques d'emboîtement dans le récit : elles sont composées par trois Anglais, puis envoyées au voyageur qui vient de rentrer de France, lequel les lit à haute voix à son ami londonien. Elles sont ensuite recopiées par celui-ci, puis envoyées en province à son correspondant, et finalement imprimées avec les autres lettres à l'attention des lecteurs. En bref, nous avons certes quelques témoignages venant de France sur les événements du Poitou, mais ces témoignages ne parviennent aux lecteurs qu'en toute fin de texte et que par l'intermédiaire de quatre récits d'Anglais (les résidents de Paris, le voyageur, le londonien, le provincial). La parole huguenote ne se fait entendre qu'à une très lointaine distance, enchâssée dans des récits cadres dont on perd facilement le fil.

Un seul texte pourrait échapper à cette règle, A True and Faithful Narrative of the Late Barbarous Cruelties and hard Usages, exercised by the French against Protestants at Rochel (Londres, 1681). Dès la première page, on apprend qu'il s'agit de la narration d'un réfugié, arrivé à Londres le 24 septembre 1681, et le récit débute à la première personne, une exception notable parmi les autres titres. Les lecteurs n'échappent pas aux détails des tortures, mais l'auteur indique qu'il ne souhaite guère aller plus loin, les mots manquant pour décrire les sommets de l'horreur ${ }^{69}$. À la fin du récit, on apprend toutefois qu'il n'a pas directement connu les souffrances, même s'il est né en France (il ne donne pas son nom, mais signe 'P.L.') : il se contente de rapporter ce qu'il lui a été communiqué par l'un des rescapés de La Rochelleincidemment un lieu de mémoire problématique pour les rapports entre l'Angleterre et les protestants français. La fin du texte est consacrée à un panégyrique de Charles II et de la nation anglaise, digne d'un Lombard, et la signature est assortie d'une profession de foi anglicane, ' $A$ Born French, but desirous to dy An English Protestant, P.L.' Il ne s'agit donc pas, une fois de plus, du récit d'un exilé de 1681, mais d'un témoignage indirect qui provient d'une source favorable au gouvernement anglais.

Comment expliquer cette absence de témoignages direct en 1681, en particulier par des auteurs ou éditeurs qui furent favorables à l'immigration des huguenots, et donc peut-être à même de recueillir et de faire imprimer leurs récits ? Les ministres français déjà réfugiés en Angleterre auraient pu, eux aussi, prendre la plume pour évoquer les souffrances de leurs compatriotes. En ce qui les concerne, on peut supposer que se concilier les autorités, trouver des fonds, administrer les aides dans l'urgence, furent des tâches bien plus pressantes que la publication de récits de tortures, d'autant plus que ces derniers pouvaient contribuer à

\footnotetext{
${ }^{68}$ The Present State, pp. 26-29.

${ }^{69}$ A True and Faithful Narrative, sig. A2r.
} 
envenimer la situation en France. On peut toutefois aussi imputer le refus d'une parole directe au climat de peur précédemment évoqué : les huguenots ont peur d'offenser Charles II et donc de compromettre les aides financières, s'ils condamnent trop violemment les événements de France (même si Louis XIV était, bien évidemment, toujours lavé de tout soupçon). Mais trop peu d'études se sont penchées sur la peur des Anglais. Dans l'après 'complot papiste', alors que font rage les querelles sur le catholicisme du futur successeur au trône, la population ne guette pas les bateaux français les bras chargés de vivres, de couvertures et de Bibles, avec aux lèvres la promesse d'un travail dans le port. Avant de se prononcer, ne serait-ce que sur le protestantisme de ces exilés, les Anglais attendent des intermédiaires, mais des intermédiaires qu'ils connaissent et auxquels ils font confiance. L'arrivée des huguenots est perçue à travers les explications de leurs juristes, les informations de leurs journalistes, les gestes de leur roi, les requêtes de leurs évêques, les prêches de leurs ministres et ils attendent des signes d'obéissance (ou tout au moins de non-résistance) des exilés au monarque et à l'anglicanisme. En ce sens, même - et peut-être surtout—les auteurs les plus dévoués à la cause huguenote ne pensaient pas forcément que la meilleure façon d'aider les réfugiés étaient de leur laisser prendre le contrôle de la représentation de leur propre exil.

Nous pouvons donc tirer certaines conclusions de ce survol : malgré la variété des supports, les récits de persécutions présentent tous un caractère légal, donc prétendument objectif. D'une part, par souci d'information du lectorat anglais à qui l'on rappelle sans cesse la teneur de l'Édit de Nantes pour qu'il puisse comprendre les persécutions de 1681. D'autre part, les textes légaux contrecarrent les récits les plus sensationnels des souffrances et imitent en quelque sorte les deux aspects des événements français : la répression officielle et l'envoi des dragons. Enfin, la distance entre les narrateurs et l'objet de leur récit est toujours évidente. Lorsqu'il est possible de déterminer les sympathies des auteurs, traducteurs ou éditeurs, on constate que les Whigs n'hésitent pas à présenter la dragonnade dans toute son horreur pour appeler à une union de tous les protestants et lancer un grand élan de solidarité parmi la population anglaise. Cette stratégie se révéla être un échec relatif.

\section{La dragonnade et la querelle entre Whigs et Tories}

L'un des adversaires de l'immigration huguenote a pu écrire: 'I hope I may without offence, take the Liberty at least to enquire whether the Persecution of Dissenters in France be so really dreadful, and the motives of the flocking over of such vast Numbers of them hither, be so pureful spiritual as is pretended... whatever either they themselves avouched, or True Protestant News- 
mungers invented for them ${ }^{, 70}$. Il faut nous attarder sur le vocabulaire employé : les huguenots deviennent les 'dissidents' de France, le terme étant réservé en Angleterre aux ministres qui refusaient de se conformer à l'Acte d'Uniformité de 1662. D'autre part, les 'True Protestant News-Mungers' sont les journalistes whigs qui ont adopté le terme 'vrais protestants' pour se démarquer de leurs adversaires tories et se présenter en véritables défenseurs du protestantisme international ${ }^{71}$. Ils sont directement accusés d'avoir 'inventé' les souffrances huguenotes. Les huguenots sont à présents considérés comme des alliés des factions les plus radicales du royaume.

La dragonnade poitevine arrivait dans un contexte pour le moins troublé en Angleterre. Les rumeurs commencèrent à poindre dans la presse à partir du mois de juillet 1681 , donc quatre mois à peine après la dissolution du parlement d'Oxford qui avait mis un terme à l'espoir d'exclure du trône par voie parlementaire le catholique duc d'York, le futur Jacques II $^{72}$. Dans les mois qui suivirent, les Tories regagnèrent le contrôle de la presse, emmenés par le flamboyant pamphlétaire et journaliste Roger L'Estrange. Les Whigs entrèrent alors dans une traversée du désert, qui atteint son nadir avec la condamnation à mort de Stephen College accusé d'avoir voulu s'emparer de la personne du roi lors du Parlement d'Oxford en mars 1681 et l'arrestation, en juillet, de la figure emblématique du mouvement, le Comte de Shaftesbury. Jurieu dira de lui, 'il a peut-estre l'ame un peu trop republicaine pour estre habitant d'une Monarchie : mais nous ne le croyons pas capable des lâchetez dont on l'accuse, ${ }^{, 73}$.

Si on accepte que les Whigs étaient favorables à l'immigration des huguenots parce qu'ils présageaient une entente possible avec les non-conformistes, les Tories étaient dans une position plus difficile. Les plus timorés pouvaient véhiculer l'image du huguenot comme dissident ou crypto-catholique profiteur venant saper les institutions ecclésiastiques et économiques du royaume. La plupart, cependant, choisirent d'autres tactiques pour tenter de s'approprier les retombées positives de l'exil religieux : insister sur l'accueil des protestants par Charles II, ce qui confirmait son protestantisme (c'est ainsi que le Français 'P.L.' peut livrer un récit de dragonnade qui conclut sur sa volonté de mourir 'en protestant anglais'), tenter une alliance avec les huguenots, à condition de pouvoir persuader leurs ministres de se prononcer clairement en faveur de l'anglicanisme et de son système épiscopal.

Sans entrer dans la complexité de la presse à la Restauration, on peut brièvement rappeler que l'on a affaire à différents types de publications. D'abord une seule publication 'officielle', c'est-à-dire contrôlée par le Secrétaire d'État, The London Gazette; puis des 'journaux',

\footnotetext{
${ }^{70}$ Actes of the General Assembly, sig. A2v.

${ }^{71}$ Voir par exemple, Schwoerer, pp. 155-156.

${ }^{72}$ Pour Narcissus Luttrell les persécutions ont débuté environ un an auparavant, le 4 août 1680 : 'About this time the king of France began to persecute the Hugonots in his kingdomes' (Luttrell, vol. 1, p. 55).

${ }^{73}$ Jurieu, Derniers Efforts, II, p. 126.
} 
essentiellement whigs, c'est-à-dire quelques feuilles dont la fonction était de présenter les nouvelles ayant trait à la politique intérieure ou extérieure, avec un fort parti-pris politique variant selon les journalistes. Viennent ensuite des textes que les auteurs nommaient pamphlets mais qui se présentaient souvent comme des journaux et qu'un historien a donc nommé 'pamphlets périodiques' (des publications hebdomadaires ou bi-hebdomadaires, séparées en colonnes, se terminant par des publicités ou annonces officielles, comme par exemple The Observator de Roger L'Estrange $)^{74}$. Il ne s'agit ici pas véritablement de ce qu'on pourrait appeler la 'presse d'information', mais de commentaires (souvent sous forme de dialogues) de personnalités charismatiques et controversées sur tel ou tel événement de la semaine.

La première observation concerne The London Gazette. L'essentiel de la publication est constitué par les décrets du royaume (une sorte de 'journal officiel' anglais) et les nouvelles étrangères, fournies au Secrétaire d'État par ses correspondants étrangers ou prises dans les gazettes de France, de Belgique et de Hollande ${ }^{75}$. Etant donné le contrôle par le gouvernement (et la volonté de ne rien inclure dans The Gazette qui soit trop controversé), il n'y est fait aucune mention de la dragonnade entre juin et novembre 1681. Le journal se contente d'attirer l'attention des lecteurs sur les 'sévérités' à l'encontre des protestants français à seulement deux reprises (les deux ayant trait au sort des enfants huguenots, signe, là encore, de l'impact de la déclaration de juillet en France, comme à l'étranger) ${ }^{76}$. The London Gazette peut être accusée de minimiser le danger afin de ne pas choquer les sensibilités de Louis et du clergé catholique et éviter ainsi d'envenimer les relations entre protestants et catholiques anglais, ce qui pourrait provoquer une alliance entre anglicans et non-conformistes.

Dans la catégorie des journaux avec un parti pris politique, on peut citer celui de Thomas Benskins, The Domestick Intelligence: Or News both From City and Country Impartially Related. Voici l'une de ses descriptions de la dragonnade, qui mentionne viols et meurtres, là encore sans aucune preuve :

Priests and Jesuits... Triumph over them with all manner of Insolencies by Imprisonings, Confiscations, Rapes, Murtherers, Firing, and Demolishing, forcing Women from their Husbands, and Children from their Parents, Burning Houses, Taxation Extraordinary, Imposts and such barbarous Severities the worst of the Heathen Emperours scarce ever used against the Christians ${ }^{77}$.

The Impartiall Mercury, s'intéresse, quant à lui, au détail des tortures physiques, nous l'avons vu, mais aussi au nombre des réfugiés. Le 29 juillet, 28 ministres ont déjà quitté la

\footnotetext{
${ }^{74}$ Pour la typologie, voir Peter Fraser, The Intelligence of the Secretaries of State and their Monoply of Licensed News, 1600-1688 (Cambridge, 1956), pp. 122, 167.

${ }^{75}$ Ibid., pp. 51-56. Voir également, James Sutherland, The Restoration Newspaper and its Development (1986; Cambridge, 2004), pp. 123-145.

${ }^{76} \mathrm{~N}^{\circ} 1634$ (du 14 au 18 juillet 1681 ), $\mathrm{n}^{\circ} 1637$ (du 25 au 28 juillet 1681 ).

${ }^{77} \mathrm{~N}^{\circ} 20$ (du 28 juillet au $1^{\text {er }}$ août 1681). Voir aussi les numéros $n^{\circ} 22,23,25,27,28,29$.
} 
France $^{78}$. Quelques jours plus tard, ils sont rejoints par 322 réfugiés, suivis d'“environ 300' autres, hommes, femmes et enfants qui débarquent à Londres début septembre ${ }^{79}$. La semaine suivante, trois vaisseaux arrivent avec à bord environ 100 personnes, des marins et leur famille. Enfin début octobre, ce sont les 20 personnes en provenance de Bordeaux qui se présentent dans l'état d'épuisement physique précédemment décrit. Langley Curtis, pour la même semaine du 29 juillet, avance des statistiques un peu différentes de celles de son confrère : dans son True Protestant Mercury : Or, Occurrences Foreign and Domestick, il est question de 500 personnes qui auraient quitté la France pour l'Angleterre en une seule semaine, essentiellement des artisans (Handycraft men) ${ }^{80}$. Comme Care et Benskins, Langley Curtis rapporte aussi dans presque tous les numéros de son journal parus pendant l'été la violence des persécutions, ainsi que les efforts des autorités anglaises pour accueillir au mieux les réfugiés ${ }^{81}$.

Le farouche Curtis se fait même ici étrangement obséquieux : les autorités municipales sont chaleureusement remerciées pour leurs efforts, mais aussi Sa Majesté dont les nombreuses 'déclarations' et 'résolutions' remplissent de joie les malheureux qui débarquent sur ses côtes ${ }^{82}$. Il est peu probable, étant donné son passé de dissident notoire et ses emprisonnements successifs, que Curtis se soit soudain laissé aller à vanter la générosité de son roi sans arrièrepensées. Il semblerait qu'il faille voir dans l'insistance avec laquelle il rappelle ici la bonté de Charles II une volonté de ne pas aliéner le monarque envers les huguenots, alors que les soupçons de presbytérianisme pesaient déjà fortement sur eux. Le meilleur moyen de leur venir en aide était encore sans doute de ne pas paraître entièrement de leur côté.

Il nous faut donc tenter de démêler les motifs des Whigs qui, dans l'ensemble, présentent la dragonnade de façon très crue, tout en insistant sur la générosité des autorités. D’une part, les Whigs virent sans doute dans les attaques menées contre les protestants français à l'été 1681, une occasion presque inespérée de retourner la situation à leur avantage et de peindre le danger que représenterait pour l'Angleterre un monarque qui ne serait pas de même confession que ses sujets. Benskins, Janeway, Care et Curtis n'hésitèrent guère à dépeindre les souffrances des protestants français semaine après semaine pour tenter de convaincre leurs lecteurs du bienfondé d'une politique exclusionniste et pour appeler à une union de tous les protestants. En même temps, il est fort probable que ces grands acteurs du monde de l'édition se soient rendu compte qu'ils jouaient un jeu dangereux : d'une part, ils risquaient d'envenimer la situation en France; d'autre part, leur soutien inconditionnel aux huguenots pouvait être contre-productif

\footnotetext{
${ }^{78}$ The True Impartiall Mercury, $\mathrm{n}^{\circ} 27$ (du 22 au 26 juillet 1681), $\mathrm{n}^{\circ} 28$ (du 19 au 26 juillet 1681).

${ }^{79}$ Ibid., $\mathrm{n}^{\circ} 31$ (du 5 au 9 août 1681) et $\mathrm{n}^{\circ} 41$ (du 9 au 13 septembre 1681).

${ }^{80}$ The True Protestant Mercury, $\mathrm{n}^{\circ} 58$ (du 23 au 27 juillet 1681).

${ }^{81}$ Voir par exemple, Ibid., n ${ }^{\circ} 59$ (du 27 au 30 juillet 1681), n 60 (du 30 juillet au 3 août 1681), nº 62 (du 10 au 13 août 1681), $\mathrm{n}^{\circ} 67$ (du 24 au 27 août 1681), nº 68 (du 27 au 30 août 1681).

${ }^{82}$ Ibid., $\mathrm{n}^{\circ} 80$ (du 8 au 12 octobre 1681).
} 
puisque les Français étaient déjà soupçonnés, avant même de fouler le sol anglais, d'être hostiles à la fois aux Stuarts et à l'anglicanisme. Il fallait donc trouver un subtil équilibre entre pathos et distance critique, mais il nous semble que les journalistes ont dans l'ensemble échoué, en faisant trop souvent basculer les comptes-rendus dans l'horreur.

Enfin, il nous faut examiner les 'pamphlets' dont le parti-pris est encore plus évident. Dès décembre 1680, Henry Care l'auteur du plus célèbre pamphlet whig (publié par Curtis jusqu'en août 1682), The Weekly Pacquet of Advice from Rome: Or, The History of Popery, avait dénoncé les persécutions des huguenots ${ }^{83}$. En juillet, au beau milieu de la dragonnade, l'un des personnages du dialogue de Care se demande si l'on ne devrait pas finalement prendre les huguenots en pitié. La réponse ironique de son interlocuteur est sans appel : 'Pitty them! What sure you wo'nt turn Fool in your Old Age? Those Hugenots are Presbyterians, man! And errant dissenters ${ }^{84}$. Care insinue que les Tories reprochent maintenant aux huguenots de s'être alliés avec les factions les plus radicales du royaume. L'accueil des réfugiés est donc en fait freiné par les soupçons de dissidence religieuse, un huguenot étant facilement surnommé 'A Traiterous Villain of a Calvinistical Hugenote ${ }^{, 85}$.

Care va plus loin et accuse son ennemi tory, Roger L'Estrange, d'encourager le climat de méfiance à l'encontre des huguenots, voire de se moquer de leurs souffrances, alors même que le roi vient de proclamer publiquement son soutien aux huguenots :

His Majesty according to his Gracious Inclinations Compassionates and orders Relief for the persecuted French Protestants. And at the same time the Observator Laughs at their Sufferings, and will needs prove them all, but Flea-bitings, because forsooth, the French King wears but one pair of Legs ${ }^{86}$.

Cette citation fait référence à un trait d'humour que L'Estrange avait cru bon d'insérer dans son Observator la semaine précédente, même s'il est aujourd'hui difficile de saisir la drôlerie de l'échange. L'Estrange s'était moqué d'une prétendue anecdote circulant dans les coffee houses selon laquelle un huguenot avait subi le supplice de la roue pour avoir refusé de désigner l'endroit où se cachaient ses enfants. L'Estrange avait rétorqué que si cela était vrai, Louis XIV ne serait pas un homme, mais un animal ${ }^{87}$. Pour ses adversaires, nier l'évidence des persécutions et faire de l'humour sur le dos des protestants français dépassaient clairement les bornes de la bienséance. Le 31 août, L'Estrange dut répondre aux accusations d'Henry Care. Selon l'Estrange, il n'était absolument pas question de se moquer de la réalité des persécutions,

\footnotetext{
${ }^{83}$ Pour Care, voir Schwoerer (pp. 44-75 pour The Weekly Pacquet et son lectorat) et Sutherland, p. 204.

${ }^{84}$ Weekly Pacquet, 3.60 (vendredi 29 juillet 1681).

${ }^{85}$ Ibid., 4.2 (30 décembre 1681).

${ }^{86}$ Ibid., 3.64 (26 août 1681). Voir aussi 3.62 (12 août 1681).

${ }^{87}$ The Observator, 1.45 (20 août 1681).
} 
mais de se moquer du traitement des prétendues 'informations' par les Whigs ${ }^{88}$. Le simple fait que les tortures les plus horribles soient rapportées par la presse whig devraient suffire à instiller le doute quant à leur véracité. Les motifs des Whigs sont limpides pour L'Estrange : en faisant en sorte d'exagérer, voire d'inventer, les souffrances des huguenots, ils tentent de déchaîner l'opinion publique anglaise contre les catholiques. Or comme les anglicans sont accusés d'être des papistes, la boucle est bouclée : les Whigs défendent les huguenots pour affaiblir l'église anglicane. Quant à L'Estrange, il ne souffle guère mot dans son propre journal du sort des protestants français.

L'Estrange reçut du renfort des auteurs du magnifique Heraclitus Ridens : At a Dialogue between Jest and Earnest, concerning the Times dont la verve rivalisait avec celle de The Observator. Heraclitus est une publication collective mais on attribue la plupart de ses numéros aux Tories Edward Rawlins et Thomas Flatman. Ces derniers, qui condamnent le trio JanewayCare-Curtis aussi violemment que L'Estrange, vont toutefois encore plus loin que leur collègue dans leurs accusations. Lassés des mensonges des Whigs, ils expliquent clairement dans le numéro du 25 octobre que ce sont en fait les Whigs qui sont à l'origine des persécutions en France :

The French King having heard of the actions of the 41 Whigs and their Principles of Rebelling, and cutting off Kings heads in order to a thorough Reformation; thought the French Protestants by their actions and writings utterly disowned the Principles ... yet the Jesuits possessing that great Monarch with the danger of suffering them to grow numerous from the deplorable Tragedy acted by the English Whigs, who will needs claim kindred, and call the Gallican Protestants Brethren ${ }^{89}$.

Là encore, la logique est claire : les Whigs sont des monarchomaques, aujourd'hui opposés à Charles II. Les Whigs et les huguenots ont une parenté profonde (kindred), ils s'appellent 'Frères' (Brethren) ; par conséquent, on ne peut guère en vouloir à Louis XIV de ne pas faire confiance à ses sujets protestants, en particulier puisqu'il est sous l'influence des jésuites. En bref, les Whigs anglais sont responsables de la dragonnade poitevine. Comme L'Estrange, il est alors hors de question pour Rawlins et Flatman d'entrer dans les détails des persécutions dans leur journal.

Les réponses des Tories aux représentations whigs de la dragonnade sont donc d'abord 'négatives', à savoir qu'ils se contentent dans un premier temps de dénoncer les manipulations de leurs ennemis politiques. Il ne faudrait pas toutefois négliger un second aspect, plus 'positif' cette fois, de la contre-attaque tory : l'enrôlement des huguenots dans le camp anglican, pour justement briser les liens de 'parenté' et de 'fratrie' entre huguenots et dissidents religieux. En

\footnotetext{
${ }^{88}$ Ibid., 1.48 (31 août 1681).

${ }^{89}$ Heraclitus Ridens, ${ }^{\circ} 39$ (25 octobre 1681).
} 
mai 1680, Edward Stillingfleet, futur évêque de Worcester, prononça un sermon qui dénonçait les schismatiques non-conformistes, The Mischief of Separation (Londres, 1680). Le sermon déclencha une telle polémique que Stillingfleet fut obligé de répondre à ses détracteurs l'année suivante dans un épais volume, The Unreasonableness of Separation (Londres, 1681). Selon Stillingfleet, il était important de ne pas laisser aux presbytériens le privilège de se proclamer frères spirituels des calvinistes du continent, des huguenots en particulier, et ce afin d'œuvrer pour une union entre tous les protestants modérés (qui laisserait la possibilité de persécuter des sectes plus radicales, pour lesquelles aucune tolérance n'était envisagée). Pour ce faire, Stillingfleet demanda à Henry Compton de lui fournir des témoignages de protestants européens prêts à déclarer ouvertement leur soutien à l'anglicanisme ${ }^{90}$. Compton tenta en vain d'entrer en contact avec certains calvinistes hollandais, par l'intermédiaire de Thomas Ken, alors aumônier de la princesse d'Orange à la Haye ${ }^{91}$. Compton obtint finalement l'aide de trois ministres français, Étienne Le Moyne, Jean-Maximilien de Baux de L'Angle et Jean Claude, qui lui adressèrent par écrit leur soutien au système épiscopal anglais. Ces lettres furent ensuite transmises par Compton à Stillingfleet qui les publia avec leur traduction anglaise, mais sans le consentement de leur auteur, à la fin de The Unreasonableness of Separation. L'incident provoqua la colère de Claude qui se sentit manipulé, voire trahi, par les prélats anglais et qui répondit sèchement à Compton, avec lequel il avait pourtant d'excellentes relations, qu'il n'avait jamais songé à soutenir Stillingfleet dans la querelle qui l'opposait aux nonconformistes $^{92}$.

Roger L'Estrange suivit, en quelque sorte, l'exemple de Stillingfleet. Au moment où, en tant que journaliste, il s'employait à dénoncer les abus des Whigs concernant la représentation de la dragonnade, il traduisait en parallèle un ouvrage anonyme français, Apologie pour les Protestans. Où l'Auteur justifie pleinement leur conduite \& leur separation de la Communion de Rome et propose des moyens faciles \& raisonnables pour une sainte et bienheureuse Reunion (Amsterdam, 1680). ${ }^{93}$ Comme Stillingfleet (qu'il mentionne), L'Estrange tente de

\footnotetext{
${ }^{90}$ Pour Compton et les huguenots, voir Nishikawa, pp. 131-177.

${ }^{91}$ Voir Bodl. MS Rawlinson C 983, f 53.

92 Edward Stillingfleet, The Unreasonableness of Separation: Or, An Impartial Account of the History, Nature, and Pleas of the Present Separation from the Communion of the Church of England... To which Several Late Letters are Annexed, Of Eminent Protestant Divines Abroad, $3^{\text {ème }}$ éd. (Londres, 1682), pp. 403-420. Pour les lettres de Claude dans lesquelles il exprime son mécontentement, voir Les Oeuvres Posthumes de Mr. Claude, 5 vols (Amsterdam, 1688-89), vol. 5, pp. 264-267. Claude Grostête de la Mothe dira plus tard que les lettres avaient pour seul motif d'encourager l'immigration huguenote; Claude Grostête de la Mothe, Correspondance Fraternelle de l'Eglise Anglicane, Avec les autres Eglises Réformées et Etrangères (La Haye, 1705), pp. 10, 12. Pour des commentaires, voir Nishikawa, pp. 142-144.

${ }^{93}$ Le titre anglais est An Apology for The Protestants: Being A full Justification of their Departure from The Church of Rome, With Fair and Practicable Proposals for A Re-union (Londres, 1681). Pour une étude complète de ce texte et des liens entre L'Estrange et Stillingfleet, voir mon chapitre, 'Roger L'Estrange and the Huguenots: Continental Protestantism and the Church of England', dans Anne Dunan-Page et Beth Lynch (eds), Roger L'Estrange and the Making of Restoration Culture (Aldershot, à paraître).
} 
démontrer que le ministre réformé qu'il traduit est un ardent défenseur de l'anglicanisme, les protestants et les catholiques pouvant se rejoindre, quelles que soient leurs différences, sur le principe de défense de la monarchie et du système épiscopal anglais. L'Estrange est obligé de jouer un double-jeu : attaquer la dragonnade comme une invention whig pour tromper l'opinion publique anglaise mais tenter en parallèle une ouverture vers les ministres français qui pourraient, à terme, contribuer à faire de l'anglicanisme le modèle du protestantisme européen. Bien évidemment, ni Stillingfleet, ni L'Estrange, ne se prononcent sur les hordes de huguenots 'profiteurs', cinquième colonne de Rome. Ils ne songent qu'à se concilier les faveurs des ministres les plus respectés, non pas tant pour favoriser l'exil des protestants, sur qui pesaient encore de lourds soupçons, que pour défendre l'anglicanisme, son roi, ses évêques et sa liturgie.

\section{Conclusion}

En 1681, le contexte politique anglais ne poussait guère à la réserve et l'on sent bien les journalistes whigs assouvir la soif des lecteurs pour les détails les plus sanguinolents avec l'énergie du désespoir. Ce parti-pris 'populiste' ne semble pas avoir très bien fonctionné. Pourtant, à l'inverse, dénoncer la dragonnade comme une invention des huguenots et de la presse whig, c'était risquer d'être accusé d'indifférence quant au sort du protestantisme européen. Il nous semble que la grande force des Tories (et l'on pourrait ajouter de certains prélats anglicans) à ce moment précis fut d'utiliser les ressorts de la presse pour se présenter devant l'opinion publique en nobles garants de la vérité historique tout en encourageant une union des anglicans et des huguenots contre les dissidents.

Une telle attitude, que l'on retrouve chez les Tories à la L'Estrange, mais aussi chez Stillingfleet et dans une moindre mesure chez le Whig Burnet, reflétait cependant une manipulation des positions huguenotes: dans leurs écrits, les huguenots semblent être unanimement en faveur de l'épiscopalisme anglican ou au moins ne pas le désavouer. Or on connaît aujourd'hui les sympathies des huguenots pour les presbytériens avec lesquels ils partageaient l'expérience des persécutions et de la clandestinité, mais aussi une certaine méfiance à l'égard du pouvoir épiscopal et de la généralisation du rite anglican, imposé sans aucune concession.

Un grand travail reste à faire sur la distinction entre l'exil des ministres et l'exil des travailleurs et artisans, qui n'étaient pas perçus de la même façon, du moins par la population anglaise. Reste que les événements de 1681 faisaient du protestantisme français un enjeu de taille dans les querelles internes de l'Angleterre. Des voix anonymes (peut-être tories ?) tentaient de faire passer les Français pour des rebelles papistes qui allaient mettre le royaume à 
feu et à sang ou pour des dissidents prêts à renverser le système épiscopal anglican. Ouvertement, tout le monde courtisait pourtant les huguenots: les Tories, parce que les conditions d'accueil généreuses de Charles II le libéraient du même coup des accusations de ‘tiédeur' envers les protestants et parce qu'au moins certains huguenots embrassaient sans scrupules de conscience l'anglicanisme; les Whigs, au contraire, parce qu'ils espéraient une alliance protestante contre Rome, un terrain d'entente entre dissidents modérés et huguenots (d'ailleurs parfois au mépris des protestants les plus radicaux).

Ce travail appelle une dernière remarque qui concerne la façon dont les huguenots ont été représentés. Il est apparu que des rumeurs circulaient non seulement sur les dangers économiques et religieux de leur arrivée en Angleterre, mais également sur leur mauvaise foi. Malgré la présence de communautés huguenotes en Angleterre depuis près de 150 ans, il semblerait que les Anglais aient eu besoin de la médiation de leurs propres auteurs avant de croire, ou de ne pas croire, les récits et témoignages directs de ceux qui accostaient sur leurs rivages. Dans le contexte de la Crise de l'Exclusion, un exilé calviniste devait pouvoir apporter la preuve de ses dires, malgré le soutien du monarque et des factions anti-catholiques pour qui il symbolisait le rêve d'une internationale protestante. Il faut donc appeler à une nouvelle étude qui analyserait cette fois les moyens par lesquels les huguenots tentèrent de faire entendre leur voix au milieu de ce chœur d'Anglais qui leur a parfois (mais pas toujours de façon mal intentionnée) usurpé le droit de représenter leur propre exil. 